\title{
ON A THEOREM OF KLEE
}

\author{
R. D. ANDERSON
}

In 1953 and 1956, Klee [3], [4] proved that for $E$ any infinitedimensional normed linear space and $K$ any compact subset of $E$, $E \backslash K$ is homeomorphic to $E$. Klee's argument used sequences of bounded convex sets. In [5], Lin has some extensions of Klee's results using modifications of his methods. In this paper we give a short and elementary proof of a somewhat more general result ${ }^{1}$ using only simple set-theoretic properties.

A space $S$ is said to be an $\alpha$-space provided

(1) $S$ is an infinite-dimensional topological linear space, i.e., an infinite dimensional real vector space with a Hausdorff topology in which vector addition and scalar multiplication are jointly continuous,

(2) $S$ has a Schauder basis, i.e., a sequence $\left\{x_{i}\right\}_{i>0}$ of elements of $S$ such that for each $s \in S$ there is a unique sequence of scalars $\left\{a_{i}\right\}$ with $s=\sum_{i=1}^{\infty} a_{i} x_{i}$ (convergence being in the topology of $S$ ) such that the function $f_{i}$ defined by $f_{i}(s)=a_{i}$ is continuous for each $i$, and

(3) there is a neighborhood $U$ of the origin such that the elements $\left\{x_{i}\right\}$ of the Schauder basis above are not in $U$.

Henceforth, all spaces under discussion are to be $\alpha$-spaces.

For each $i$, let $M_{i}$ denote the product of $i$ copies of the reals with usual distance function $d_{i}$ referring to distance between points, between a point and a set or between two sets. Let $f_{i}$ be as defined in condition (2) of the definition of an $\alpha$-space and let $g_{i}$ be the map of $S$ onto $M_{\imath}$ defined by $g_{i}(s)=\left(f_{1}(s), f_{2}(s), \cdots, f_{i}(s)\right)$. Since, by hypothesis, $f_{i}$ is continuous (for each $i$ ), then so is $g_{j}$ for each $j$.

A set $K \subset S$ is said to be projectible provided

(1) $K$ is closed,

(2) for any $p \in S \backslash K$, there is a $j$ such that $g_{j}(p)$ is not an element of the closure of $g_{j}(K)$, and

(3) there exist infinitely many $i$ such that $f_{i}(K)$ is bounded above or below.

The proof of the following lemma is trivial and is therefore omitted.

Presented to the Society, November 21, 1964; received by the editors March 20, 1965.

${ }^{1}$ I am indebted to V. L. Klee and to J. R. Retherford for helpful suggestions leading to the present form of the main theorem (which was originally conceived as a theorem about $l_{p}$ spaces). 
Lemma. Let $S$ be an $\alpha$-space, $s \in S, K$ be a projectible subset of $S$, and let $g_{i}(s)$ be a point of $M_{i}$ not in the closure of $g_{i}(K)$. Then, for each $j>i, d_{j}\left(g_{j}(s), g_{j}(K)\right) \geqq d_{i}\left(g_{i}(s), g_{i}(K)\right)>0$.

Theorem. Let $S$ be an $\alpha$-space and $K$ be a projectible subset of $S$. Then $S \backslash K$ is homeomorphic to $S$.

Proof. Without loss of generality, we may let $\left\{n_{i}\right\}_{i>0}$ be a sequence of integers such that, for each $i>0, n_{i}>i$ and $f_{n_{i}}(K)$ is bounded below by $z_{n_{i}}$. For each $i>0$, let $U_{i}$ be the $\left(1 / 2^{i}\right)$-neighborhood of $g_{i}(K)$ in $M_{i}$.

For each $i \geqq 1$, let $h_{i}$ be the homeomorphism of $S$ onto itself such that (1) for each $q \in S$ and each $m \neq n_{i}, f_{m}\left(h_{i}(q)\right)=f_{m}(q)$ and (2) for each $q \in S, f_{n_{i}}\left(h_{i}(q)\right)=f_{n_{i}}(q)+2 r_{q, i}\left(\left|z_{n_{i}}\right|+i\right)$ where

$$
r_{q, i}=\min \left[\frac{d_{i}\left(g_{i}(q), M_{i} \backslash U_{i}\right)}{d_{i}\left(g_{i}(K), M_{i} \backslash U_{i}\right)}, 1\right] .
$$

Let $h$ be defined as follows:

$$
\begin{aligned}
\text { for } j \notin\left\{n_{i}\right\}, f_{j}(h(s)) & =f_{j}(s) \\
\text { for any } i>0, f_{n_{i}}(h(s)) & =f_{n_{i}}\left(h_{i}(s)\right) .
\end{aligned}
$$

Then $h$ is a homeomorphism of $S \backslash K$ on to $S$ as desired and as we shall verify.

Consider $q \in S \backslash K$. By condition 2 of projectibility and the Lemma, there is a neighborhood $V$ of $q$ such that $g_{i}(V) \subset M_{i} \backslash U_{i}$ for all but finitely many $i$ 's. Thus, for all but finitely many $i$ 's, $h_{i}$ is the identity on $V$. Hence $h(q)$ is an element of $S$ and $h$ is continuous at $q$.

Let $\pi_{i}$ be a homeomorphism defined coordinatewise as follows:

$$
\begin{aligned}
& \text { for } j \leqq i, \quad f_{n_{j}}\left(\pi_{i}(x)\right)=f_{n_{j}}\left(h_{j}(x)\right) \\
& \text { for } k \notin\left\{n_{j}\right\}_{j=1}^{i}, \quad f_{k}\left(\pi_{i}(x)\right)=f_{k}(x) .
\end{aligned}
$$

We note that for $j>0, h_{j}$ is the identity except on $g_{j}{ }^{-1}\left(U_{j}\right)$. Also $g_{j}^{-1}\left(U_{j}\right) \supset g_{j+1}^{-1}\left(U_{j+1}\right)$. Thus $\left(\pi_{j+1} \pi_{j}^{-1}\right)$ is the identity except on $\pi_{j}\left(g_{j+1}^{-1}\left(U_{j+1}\right)\right)$ since $\pi_{j+1}$ acts in the same way as $\pi_{j}$ except on $\pi_{j}\left(g_{j+1}^{-1}\left(U_{j+1}\right)\right)$.

Clearly by considering successive coordinates, $h$ may be regarded as- $-\left(\pi_{j+1} \pi_{j}^{-1}\right)-\left(\pi_{3} \pi_{2}^{-1}\right)\left(\pi_{2} \pi_{1}^{-1}\right) \pi_{1}$ and for each $i, \pi_{i}$ is the product of the first $i$ indicated factors from the right. Now we think of the effects of these factors starting from the right. Let $i U$ denote the set of products of the scalar $i$ and the elements of the set $U$ of condition (3) of the $\alpha$-space definition. 
First $\pi_{1}$ moves $g_{2}{ }^{-1}\left(U_{2}\right)$ outside of $1 U$ in the $n_{1}$ direction. Thus $\left(\pi_{2} \pi_{1}^{-1}\right)$ is the identity on $1 U$. But $\left(\pi_{2} \pi_{1}^{-1}\right)$ moves $\pi_{1}\left(g_{3}^{-1}\left(U_{3}\right)\right)$ outside of $2 U$ in the $n_{2}$ direction. Thus $\left(\pi_{3} \pi_{2}^{-1}\right)$ is the identity on $2 U$. Inductively, $\left(\pi_{i+1} \pi_{i}^{-1}\right)$ is the identity on $i U$. But since, for each $i$ and each $j>0,\left(\pi_{i+j} \pi_{i+j-1}^{-1}\right)$ is the identity on $i U$, then on $i U, h^{-1}$ may be considered to be defined as

$$
\left[\left(\pi_{i} \pi_{i-1}^{-1}\right)\left(\cdots\left(\pi_{2} \pi_{1}^{-1}\right) \pi_{1}\right)\right]^{-1} .
$$

Hence since $S=\cup_{i>0} i U, h^{-1}$ is defined and continuous on $S$ and $h$ is a homeomorphism of $S \backslash K$ on to $S$.

It is clear that any Banach space with a basis is an $\alpha$-space (for, without loss of generality, we may assume that the basis elements all have norm 1). Thus for each $p \geq 1, l_{p}$ is an $\alpha$-space. It is not hard to see that all $l_{p}$ spaces for $0<p<1$ are also $\alpha$-spaces. In [6], it is shown that various topological linear spaces including some nonmetrizable ones satisfy conditions guaranteeing that they are $\alpha$-spaces. On the other hand, the countable infinite product $s$ of lines as a topological linear space is not an $\alpha$-space (the set $U$ does not exist). The argument of this paper does not work for this type of space. However, in [1] the author shows by a different argument that any countable union of compact sets or even sets comparable to projectible sets may be deleted from $s$ without changing its topological character. Since $l_{2}$ is homeomorphic to $s,[2], l_{2}$ also can lose an arbitrary countable union of compact sets without changing its topological character. Indeed, Klee's argument [3] can be easily modified to show that for $K$ any countable set of points, $l_{2} \backslash K$ is homeomorphic to $l_{2}$.

For any $\alpha$-space $S$, any compact set $K$ is projectible since, if, for each $i>0, g_{i}(q)$ is an element of the closure of $g_{i}(K)$, then as $K$ is compact, $q$ is a limit point of $K$. Clearly there are many projectible sets which are not compact. In all Banach spaces with bases, all weakly (sequentially) compact sets are projectible.

Corollary. If $S$ is an $\alpha$-space and $K$ is a compact subset of $S$, then $S \backslash K$ is homeomorphic to $S$.

Corollary. If $S$ is a Banach space with a basis and $K$ is a weakly compact subset of $S$, then $S / K$ is homeomorphic to $S$.

\section{REFERENCES}

1. R. D. Anderson, Topological properties of the Hilbert cube and the infinite product of open intervals, Trans. Amer. Math. Soc. (to appear). 
2. - - Hilbert space is homeomorphic to the countable infinite product of lines, Bull. Amer. Math. Soc. 72 (1966), 515-519.

3. V. L. Klee, Convex bodies and periodic homeomorphism in Hilbert spaces, Trans. Amer. Math. Soc. 74 (1953), 10-43.

4. — A note on topological properties of normed linear spaces, Proc. Amer. Math. Soc. 7 (1956), 673-674.

5. Bor-Luh Lin, Two topological problems concerning infinite-dimensional normed linear spaces, Trans. Amer. Math. Soc. 114 (1965), 156-175.

6. J. R. Retherford and C. W. McArthur, Some remarks on bases in linear topological spaces, Math. Ann. 64 (1966), 38-41.

Louisiana State University

\section{A SELECTION THEOREM}

\section{E. MICHAEL ${ }^{1}$}

1. Introduction. The following theorem was proved in $[1$, Footnote 7]. (A function $\phi$ from $X$ to the collection $2^{B}$ of nonempty closed subsets of $B$ is called lower semicontinuous (=l.s.c.) if $\{x \in X: \phi(x) \cap V \neq \varnothing\}$ is open in $X$ whenever $V$ is open in $B$, while $\Gamma_{B} A$ denotes the closed convex hull of $A$ in $B$.)

THEOREM 1.1 [1]. If $X$ is paracompact, if $B$ is a Banach space, and if $\phi: X \rightarrow 2^{B}$ is l.s.c., then there is a continuous $f: X \rightarrow Y$ such that $f(x) \in \Gamma_{B} \phi(x)$ for every $x \in X$.

As was pointed out in $[1$, p. 364], Theorem 1.1 remains true if $B$ is any complete, metrizable locally convex space, but it is generally false if $B$ is not metrizable. We can, however, prove the following generalization of Theorem 1.1.

Theorem 1.2. Let $X$ be paracompact, and $M$ a metrizable subset of a complete $^{2}$ locally convex space E. Let $\phi: X \rightarrow 2^{M}$ be l.s.c. and such that, for some metric on $M$, every $\phi(x)$ is complete. Then there exists a continuous $f: X \rightarrow E$ such that $f(x) \in \Gamma_{E} \phi(x)$ for every $x \in X$.

Theorem 1.2 was proved in [3] under the stronger assumption that $X$ is metrizable. While that was sufficient for the applications in [3], and probably for most other applications, it did not generalize Theorem 1.1, and was therefore never entirely satisfying. In this

Received by the editors May 23, 1966.

1 Supported by NSF Grant 11-5020.

${ }^{2}$ It suffices if $\Gamma_{E} K$ is compact for every compact $K \subset M$. 